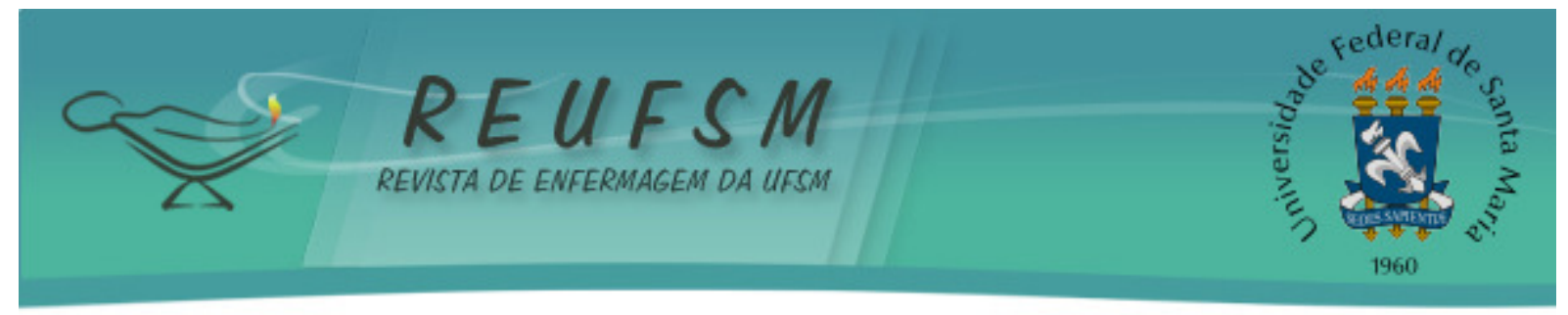

\title{
ARTIGO ORIGINAL ESTILO DE VIDA E SAÚDE DE DOCENTES DE UMA INSTITUIÇÃO DE ENSINO PÚBLICA
}

\author{
LIFESTYLE AND HEALTH OF TEACHERS OF A PUBLIC EDUCATION \\ INSTITUTION
}

ESTILO DE VIDA Y LA SALUD DE DOCENTES DE UNA INSTITUCIÓN DE ENSE NANZA PÚBLICA

\author{
Renata Cristina da Penha Silveira ${ }^{1}$ \\ Isabely Karoline da Silva Ribeiro ${ }^{2}$ \\ Graziela Silveira Teixeira ${ }^{3}$ \\ Lidiane Naiara Teixeira ${ }^{4}$ \\ Paulo Henrique Alves Souza
}

Doi: $10.5902 / 2179769224713$

RESUMO: Objetivo: identificar o estilo de vida e saúde de docentes de uma instituição de ensino pública. Método: estudo quantitativo, descritivo, correlacional. A amostra foi composta por 82 docentes dos cursos de Bioquímica, Enfermagem, Farmácia e Medicina. Utilizou-se um questionário semiestruturado para a caracterização do perfil sóciodemográfico e laboral; escala validada do Estilo de Vida Fantástico. Resultados: 65,9\% mulheres, 74,4\% doutores, $58,3 \%$ relataram estilo de vida muito bom, $24,4 \%$ afastaram devido a doença; $68,2 \%$ dos docentes com estilo de vida muito bom não fazia uso de medicação diária, ao passo que para aqueles que faziam uso de medicação diária, o estilo de vida muito bom caiu para 42,9\%. Conclusão: Há necessidade de criação de políticas públicas de saúde que visem a promoção à saúde no ambiente universitário e a produção de novos estudos acerca das alterações de saúde nos docente e suas repercussões no estilo de vida.

Descritores: Docentes; Universidades; Saúde do trabalhador; Saúde pública; Estilo de vida; Enfermagem.

ABSTRACT: Aim: to identify the lifestyle and health of teachers of a public educational institution. Method: quantitative, descriptive, correlational study. The sample consisted of 82 professors of Biochemistry, Nursing, Pharmacy and Medicine. A semi-structured questionnaire was used to characterize the socio-demographic and work profile; Validated scale of the Fantastic Lifestyle. Results: $65.9 \%$ women, $74.4 \%$ doctors, $58.3 \%$ reported a very good lifestyle, $24.4 \%$ withdrawn due to illness; $68.2 \%$ of teachers with very good lifestyle did not use

\footnotetext{
${ }^{1}$ Enfermeira. Doutora. Professora Associada da Universidade Federal de São João Del Rei (UFSJ), Campus Centro-Oeste Dona Lindu. Divinópolis-MG, Brasil, renatasilveira@ufsj.edu.br, renatacps@hotmail.com

${ }^{2}$ Acadêmica de Enfermagem na Universidade Federal de São João Del Rei (UFSJ), Campus Centro-Oeste Dona Lindu. Divinópolis-MG, Brasil, isabelykaroline@ hotmail.com

${ }^{3}$ Enfermeira. Mestranda pelo Programa de Mestrado Acadêmico em Enfermagem da Universidade Federal de São João Del Rei (UFSJ), Campus Centro-Oeste Dona Lindu. Divinópolis-MG, Brasil, ziziteixeira@hotmail.com

${ }^{4}$ Acadêmica de Enfermagem na Universidade Federal de São João Del Rei (UFSJ), Campus Centro-Oeste Dona Lindu. Divinópolis-MG, Brasil, lidint@ hotmail.com (bolsista de Iniciação Científica- PIBIC UFSJ/CNPq).

${ }^{5}$ Nutricionista em empresas privadas. Colaborador em projetos na Universidade Federal de São João Del Rei (UFSJ), Campus Centro-Oeste Dona Lindu. Divinópolis-MG, Brasil, spaulohenrique@ hotmail.com
} 


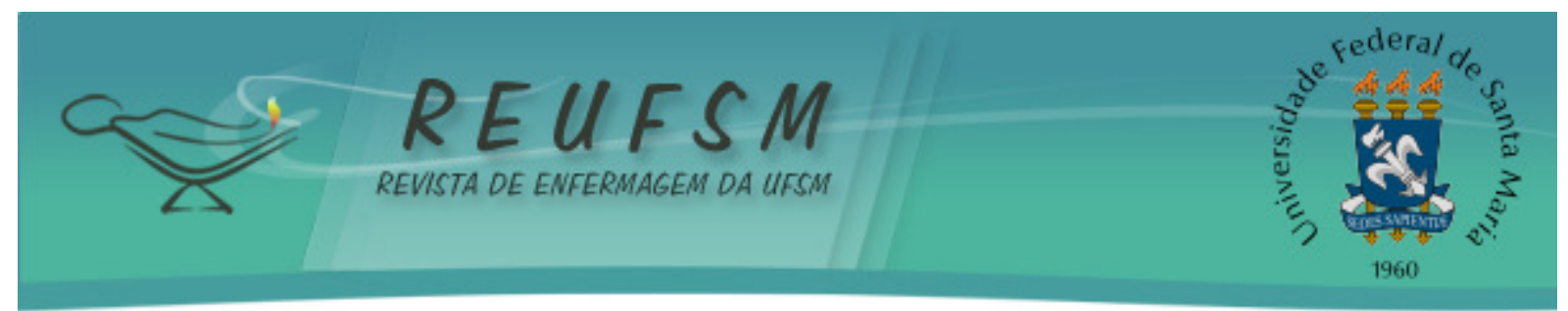

daily medication, while for those who used daily medication, the very good lifestyle dropped to 42.9\%. Conclusion: it is necessary to emphasize the need to create public health policies that aim to promote health in the university environment and the production of new studies about health changes in teachers and their repercussions on the lifestyle.

Descriptors: Faculty; Universities; Occupational health; Public health; Life style; Nursing.

RESUMEN: Objetivo: identificar el estilo de vida y la salud de los docentes de una institución de enseñanza pública. Método: estudio cuantitativo, descriptivo, correlacional. La muestra fue compuesta por 82 docentes de los cursos de Bioquímica, Enfermería, Farmacia y Medicina. Se utilizó un cuestionario semiestructurado para la caracterización del perfil socio demográfico y laboral; escala validada del Estilo de Vida Fantástico. Resultados: 65,9\% mujeres, 74,4\% doctores, 58,3\% relataron estilo de vida muy bueno, 24,4\% se alejaron debido a enfermedad; $68,2 \%$ de los docentes con estilo de vida muy bueno no hacía uso de medicación diaria, mientras, para aquellos que hacían uso de medicación diaria, el estilo de vida muy bueno cayó para 42,9\%. Conclusión: crear políticas públicas de salud que contribuyan para la promoción de la salud en el ambiente universitario y la producción de nuevos estudios sobre las alteraciones de salud de los docentes y sus repercusiones en el estilo de vida.

Descriptores: Docentes; Universidades; Salud laboral; Salud pública; Estilo de vida; Enfermería.

\section{INTRODUÇÃO}

Estudos que tratam da carreira docente desenvolveram-se a partir dos anos 1970, o que contribuiu para a melhor compreensão da trajetória profissional desses trabalhadores. A dimensão pessoal dos docentes, entretanto, tem recebido pouca atenção no contexto universitário. ${ }^{1}$

A percepção do trabalho docente no mundo globalizado reflete uma realidade social produtivista e exploradora, na qual destaca-se a precariedade e a sobrecarga de trabalho. Há necessidade de condições adequadas de materiais, estrutura e de recursos humanos, para garantir uma melhor qualidade laboral e uma vida saudável, para favorecer a saúde do trabalhador docente. ${ }^{2}$

O trabalho docente está muito além da função de ensino e produção de conhecimentos. Os docentes são cobrados a participarem de produções e publicações científicas, desenvolvimento de projetos de pesquisa, ensino, extensão, reuniões colegiadas, acompanhamentos de processos e funções burocráticas. Tais exigências em sua atuação acadêmica podem influenciar negativamente no seu estilo de vida em diferentes aspectos. ${ }^{3}$

O estilo de vida de maneira geral é baseado nas condições de vida de um indivíduo, assim, em um sentido mais amplo, envolve as condutas determinadas por fatores socioculturais e características individuais. Um estilo de vida considerado saudável sugere condutas e hábitos que influenciam a saúde de forma positiva. ${ }^{4}$ 


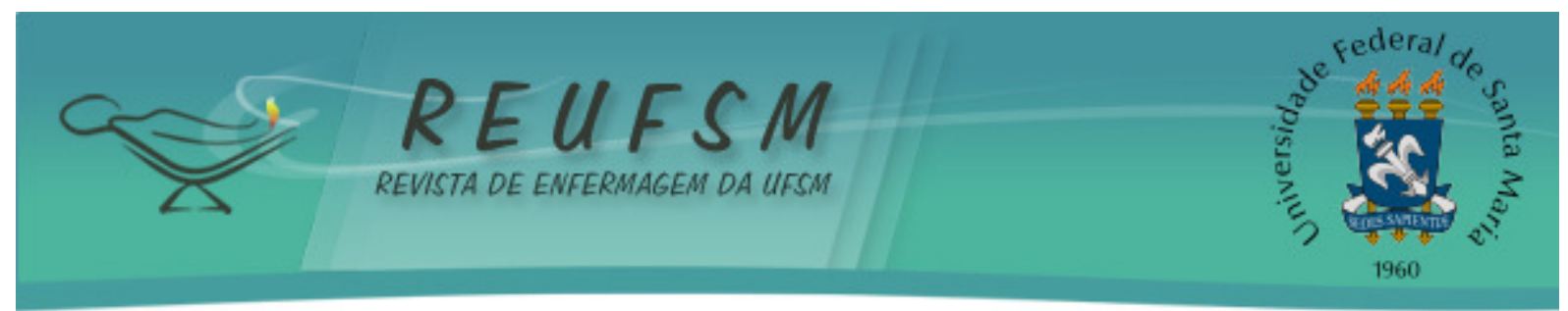

O nível de estresse nos docentes também pode influenciar na QV e no trabalho. Dentre os estressores, destacam-se situações, tais como, pouco tempo para cuidar da saúde, infraestrutura precária, sobrecarga de trabalho (aula, produção científica), dificuldade de conciliar compromissos como casa, família e trabalho, entre outros. ${ }^{5}$

Percebe-se uma escassez na literatura de investigações que tratam do estilo de vida e saúde de docentes e que foram realizados com profissionais de instituições de ensino que estão fora dos grandes centros urbanos. ${ }^{6,7}$ Nesta perspectiva, o presente estudo tem por objetivo identificar o estilo de vida e saúde de docentes de uma instituição de ensino pública.

\section{MÉTODO}

Trata-se de um estudo com abordagem quantitativa, descritivo, transversal, realizado em instituição de ensino pública localizada em município do Centro Oeste do Estado de Minas Gerais.

A população do estudo foi constituída por 151 docentes dos cursos de Enfermagem, Farmácia, Bioquímica e Medicina. A amostragem foi feita por conveniência, após convite via e-mail dirigido a professores que aglutinava o conjunto de cursos de saúde da instituição da pesquisa. Todos os docentes foram convidados a participar via e-mail institucional e após, via e-mail pessoal em quatro chamadas. Critérios de inclusão: docente efetivo; não estar de licença ou afastados das atividades acadêmicas seja por período parcial ou total. A amostra final foi composta por 82 docentes que atenderam aos critérios de inclusão.

Os dados foram obtidos por meio de um questionário semiestruturado, elaborado pelos autores, com questões referentes a caracterização do perfil demográfico e laboral. O instrumento continha questões sobre: sexo, estado civil, se possui filhos, nível de formação, vínculo de trabalho, turno de trabalho, curso que pertence, se realizava pausa durante o trabalho, se levava trabalho para a casa, se já sofreu acidente de trabalho, se já teve afastamento do trabalho por doença ou acidente de trabalho.

O Estilo de Vida foi avaliado pelo Questionário Estilo de Vida Fantástico, validado no Brasil $^{8}{ }^{8}$ que avalia estilo de vida em relação ao comportamento, uso de drogas, nutrição, que considera o comportamento no último mês e cujos dados permitem determinar a associação entre estilo de vida e saúde. As 25 questões estão dispostas na forma da escala Likert, a codificação das questões é realizada por pontos: zero para a primeira coluna, 1 para a segunda, 2 para a terceira, 3 para a quarta e 4 para a quinta coluna. As questões que só 


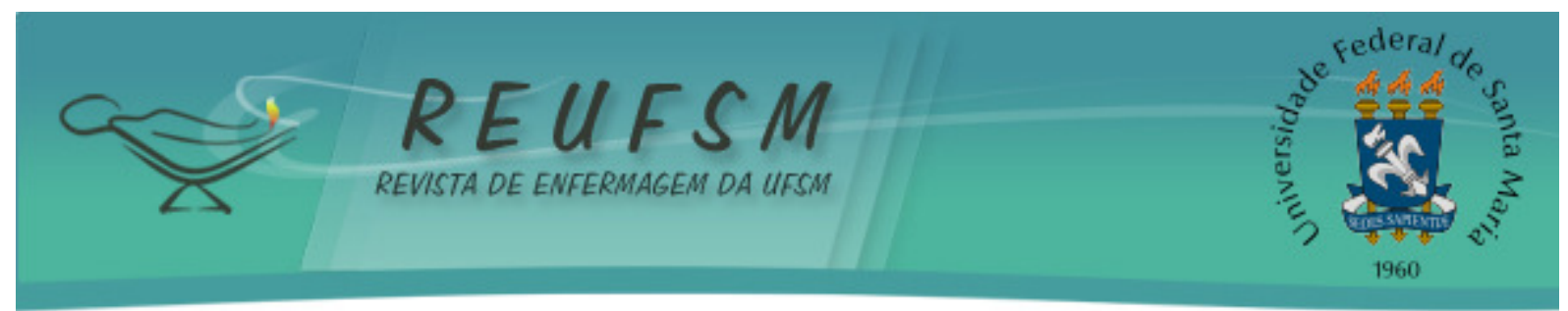

possuem duas alternativas pontuam: zero para a primeira coluna e 4 pontos para a última coluna. A soma de todos os pontos permite chegar a um escore total que classifica em cinco categorias: "excelente" (85 a 100 pontos), "muito bom" (70 a 84 pontos), "bom" (55 a 69 pontos), "regular" (35 a 54 pontos) e "necessita melhorar" (0 a 34 pontos). Quanto menor for o escore, maior será a necessidade de mudança. ${ }^{8}$

Para a preservação dos aspectos éticos da pesquisa com seres humanos, foram seguidas as disposições da Resolução 466/2012 do Conselho Nacional de Saúde (CNS). ${ }^{9}$ Para isso, o projeto de pesquisa foi submetido à análise pelo Comitê de Ética em Pesquisa com Seres Humanos da Universidade Federal de São João Del- Rei, com parecer de aprovação no 813.214.

Para a análise dos dados utilizou-se o Programa Statistical Package of Social Sciences (SPSS) versão 21.0. Foi realizada dupla digitação dos dados em planilha de Excell for Windows 7. A consistência interna das escalas foi avaliada por meio do Alfa de Cronbach. Para a construção das tabelas optou-se pela estatística descritiva, com apresentação das variáveis categóricas em frequência absoluta e relativa. Foram realizados o teste de Quiquadrado de Pearson e Correlação de Spearman, fixando-se um nível de significância de 5\%.

\section{RESULTADOS}

O alfa de Cronbach para a Escala Estilo de Vida foi de 0,67 identificando a confiabilidade interna (alfa de Cronbach substancial possui valores entre 0,8 a 0,61). ${ }^{10}$ De 151 docentes efetivos, 82 participaram do estudo sendo $42 \%$ do curso de enfermagem, $10 \%$ do curso de medicina, $16 \%$ do curso de farmácia e $14 \%$ do curso de bioquímica. A seguir, a tabela 1 exibe a caracterização desses docentes.

Tabela 1: Caracterização de uma amostra de docentes dos cursos da saúde uma instituição de ensino pública, Brasil, 2015. ( $\mathrm{N}=82)$

\begin{tabular}{lcc}
\hline \multicolumn{1}{c}{ Variáveis } & $\mathbf{N}$ & $\mathbf{( \% )}$ \\
\hline Sexo & 54 & 65,9 \\
Feminino & 28 & 34,1 \\
Masculino & & \\
Situação conjugal & 54 & 65,8 \\
$\quad$ Com companheiro & 27 & 32,9 \\
Sem companheiro & 1 & 1,3 \\
Sem resposta & & 52,4 \\
Filhos & 43 & 47,6 \\
Sim & 39 & \\
Não & & \\
\end{tabular}




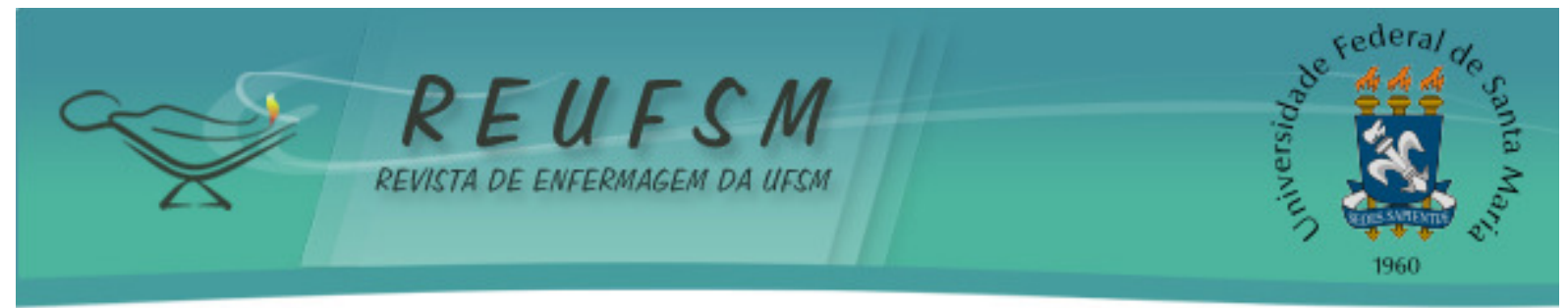

No que se relaciona à quantidade de filhos houve uma média de 1,42 filhos por docente, sendo três o número máximo de filhos/docente. A seguir, a tabela 2 apresenta as características laborais dos docentes.

Tabela 2: Caracterização do trabalho de uma amostra de docentes dos cursos da saúde uma instituição de ensino pública, Brasil, 2015. (N=82)

\begin{tabular}{|c|c|c|}
\hline Variáveis & $\mathbf{N}$ & $\%$ \\
\hline \multicolumn{3}{|l|}{ Nível de formação } \\
\hline Especialização & 1 & 1,2 \\
\hline Mestrando & 2 & 2,4 \\
\hline Mestrado completo & 2 & 2,4 \\
\hline Doutorando & 16 & 19,5 \\
\hline Doutorado completo & 61 & 74,4 \\
\hline \multicolumn{3}{|l|}{ Vinculo de trabalho } \\
\hline Dedicação Exclusiva & 71 & 86,6 \\
\hline 40 horas & 10 & 12,2 \\
\hline Sem resposta & 1 & 1,2 \\
\hline \multicolumn{3}{|c|}{ Realiza pausa durante jornada de trabalho } \\
\hline Sim & 32 & 39,0 \\
\hline Não & 20 & 24,4 \\
\hline Às vezes & 29 & 35,4 \\
\hline Sem resposta & 1 & 1,2 \\
\hline \multicolumn{3}{|l|}{ Turno de trabalho } \\
\hline Diurno & 69 & 84,1 \\
\hline Noturno & 1 & 1,2 \\
\hline Diurno/Noturno & 12 & 14,6 \\
\hline \multicolumn{3}{|l|}{ Curso ao qual pertence } \\
\hline Enfermagem & 42 & 51,1 \\
\hline Farmácia & 16 & 19,7 \\
\hline Bioquímica & 14 & 17,1 \\
\hline Medicina & 10 & 12,1 \\
\hline \multicolumn{3}{|c|}{ Leva trabalho para fazer em casa } \\
\hline Sim & 70 & 85,4 \\
\hline Não & 12 & 14,6 \\
\hline \multicolumn{3}{|c|}{ Tempo para fazer atividades do trabalho em casa } \\
\hline $1-3 h$ & 40 & 48,8 \\
\hline $4-6 h$ & 20 & 24,4 \\
\hline $7-9 \mathrm{~h}$ & 4 & 4,9 \\
\hline Sem resposta & 18 & 21,9 \\
\hline \multicolumn{3}{|c|}{ Acidente de Trabalho (AT) } \\
\hline Sim & 7 & 8,5 \\
\hline Não & 75 & 91,5 \\
\hline \multicolumn{3}{|c|}{ Afastamentos do trabalho por AT } \\
\hline Sim & 1 & 1,2 \\
\hline Não & 81 & 98,8 \\
\hline \multicolumn{3}{|c|}{ Afastamentos do trabalho devido a doença } \\
\hline Sim & 20 & 24,4 \\
\hline Não & 61 & 74,4 \\
\hline Sem resposta & 1 & 1,2 \\
\hline \multicolumn{3}{|c|}{ Sente-se curado da doença } \\
\hline Sim & 12 & 14,6 \\
\hline Não & 15 & 18,3 \\
\hline \multirow[t]{2}{*}{ Sem resposta } & 55 & 67,1 \\
\hline & 82 & 100 \\
\hline
\end{tabular}




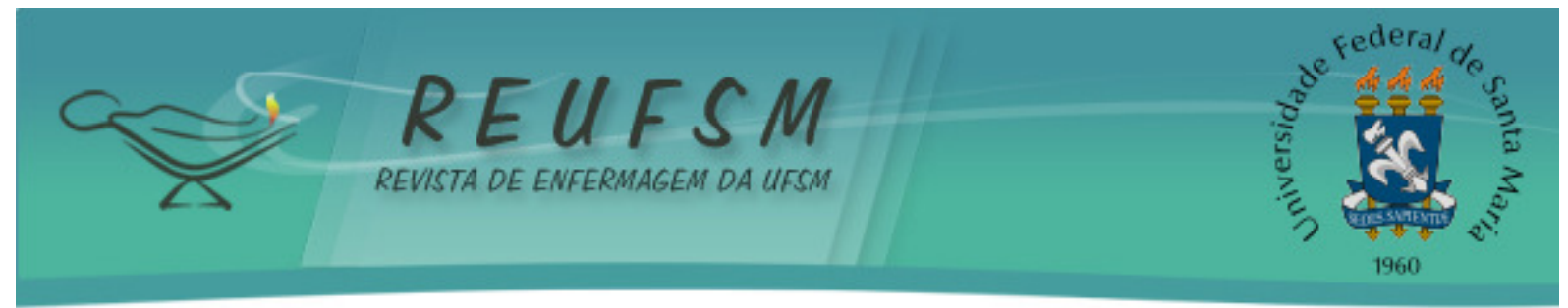

Os docentes entrevistados informaram que apesar, de realizarem pausas durante a jornada de trabalho, não existem locais apropriados para o lazer e o convívio com a comunidade universitária.

Para 63 docentes $(77,8 \%)$ o trabalho desenvolvido na universidade e serviços de saúde estão sujeitos a AT e doenças ocupacionais. Na universidade, destacam-se: risco de acidentes e doenças devido ao manuseio de material perfuro cortante, cultura de células, secreções, aulas práticas em laboratórios, gabinete docente com mobiliário inadequado, pisos escorregadios, iluminação deficiente, ausência de demarcações nos pisos. Nos serviços de saúde durante as aulas práticas com os alunos, destaca-se: o manuseio de secreções, sangue, material perfuro cortante e objetos contaminados. Em relação ao AT, os docentes se acidentaram com material perfuro cortante, queimadura de córnea, lesão no cotovelo devido ao uso excessivo do computador. Em relação aos afastamentos devido a doença destacam-se síndrome do pânico, depressão, gastroenterite, cisto nas cordas vocais, Síndrome de Burnout, dentre outros.

Para as variáveis AT e jornada de trabalho, o teste $\mathrm{X}^{2}$ evidenciou que não houve diferença estatística $(\mathrm{p}=1,00)$; assim como para as variáveis afastamento por AT e jornada de trabalho $(\mathrm{p}=1,00)$; afastamento por doença e jornada de trabalho $(\mathrm{p}=0,44)$; jornada de trabalho e uso de medicação diária $(\mathrm{p}=0,73)$.

Em relação ao estilo de vida no ambiente universitário, a tabela 3 mostra o estilo de vida dos docentes.

Tabela 3 - O Estilo de Vida de uma amostra de docentes de cursos da saúde de uma instituição de ensino pública, Brasil, 2015. (N=82)

\begin{tabular}{cccc}
\hline Variáveis & $\mathbf{N}$ & $\mathbf{( \% )}$ & $\mathbf{( \% )}$ valida \\
\hline Excelente & 09 & 11,0 & 12,5 \\
Muito bom & 42 & 51,2 & 58,3 \\
Bom & 21 & 25,6 & 29,2 \\
Sem resposta & 10 & 12,2 & 100,0 \\
Total & 82 & 100,0 & \\
\hline
\end{tabular}

Para a comparação entre o estilo de vida e o sexo, a tabela 4 apresenta os resultados.

Tabela 4 - Sexo e Estilo de Vida de uma amostra de docentes de cursos da saúde de uma instituição de ensino pública, Brasil, 2015. (N=72)

\begin{tabular}{|c|c|c|c|c|}
\hline Sexo & & Estilo de vid & & Total \\
\hline \multirow{3}{*}{ Feminino } & Bom & Muito bom & Excelente & \\
\hline & 11 & 30 & 5 & 46 \\
\hline & $23,9 \%$ & $65,2 \%$ & $10,9 \%$ & $100,0 \%$ \\
\hline \multirow[t]{2}{*}{ Masculino } & 10 & 12 & 4 & 26 \\
\hline & $38,5 \%$ & $46,2 \%$ & $15,4 \%$ & $100,0 \%$ \\
\hline \multirow[t]{2}{*}{ Total } & 21 & 42 & 9 & 72 \\
\hline & $29,2 \%$ & $58,3 \%$ & $12,5 \%$ & $100,0 \%$ \\
\hline
\end{tabular}




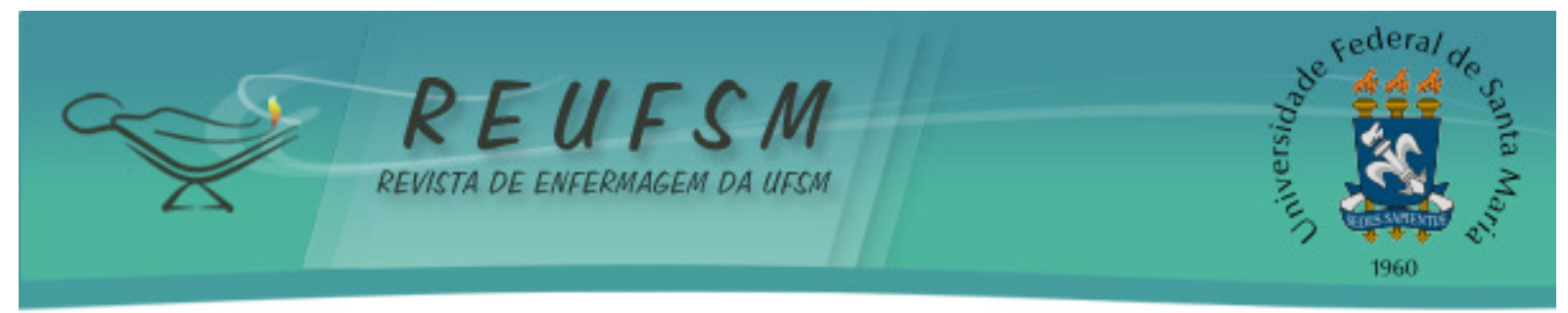

Ao realizar o Teste de $X^{2}$ de Person entre o "estilo de vida" e "sexo" $\left(X^{2}=2,51 ; p=0,29\right)$ e para as variáveis "trabalho e risco para saúde" e "sexo" $\left(X^{2}=1,29 ; p=0,26\right)$ não houve diferença significativa.

Dos docentes entrevistados, ao analisar o estilo de vida e afastamentos por doença, dos 53 docentes que não se afastaram, 11,3\% apresentaram estilo de vida excelente seguido por 58,5\% estilo de vida muito bom. Com relação aos 19 docentes que se afastaram devido a doença, 15,8\% apresentaram estilo de vida excelente, seguido por $57,9 \%$ estilo de vida muito bom, o que mostra maior porcentagem no estilo de vida muito bom para aqueles que não se afastaram do trabalho devido a doença. Ao realizar o Teste $X^{2}\left(X^{2}=0,30 ; p=0,86\right)$, não houve diferença significativa.

Para o estilo de vida e o uso de medicação diária, 68,2\% dos docentes com estilo de vida muito bom não fazia uso de medicação diária, ao passo que para aqueles que faziam uso de medicação diária, o estilo de vida muito bom caiu para $42,9 \%$. Ao realizar o teste $X^{2}$ Person $\left(X^{2}=4,54 ; p=0,1\right)$ não houve diferença estatística.

Quanto ao resultado de correlação entre nível de formação e o estilo de vida foi evidenciado correlação positiva e indica que docentes com maior titulação (doutorado) possuíam um melhor estilo de vida.

\section{DISCUSSÃO}

As pessoas passam a maior parte de suas vidas dentro das instituições de trabalho. Desde modo, é notória e inegável a relevância que o trabalho promove nas vidas dos trabalhadores. ${ }^{11}$ Deve-se promover estilo de vida saudável aos trabalhadores com destaque a ambientes de lazer, cultura, alimentação saudável e atividade física.

Investigação realizada na região norte do Brasil identificou, conforme neste estudo, a predominância do sexo feminino $(66,5 \%)$, casadas $(66,5 \%){ }^{2}$ O predomínio do sexo feminino no universo da docência é um fenômeno cadenciado por questões históricas e sociais da posição da mulher na sociedade. ${ }^{12}$

Apesar da inserção no mercado de trabalho externo ao domicílio, a mulher, em geral, acumula o trabalho familiar interno (cuidado geral de casa, dos filhos, das refeições etc.), circunstância pela qual as mulheres relatam maior estresse e desgaste físico relacionado ao trabalho, configurando-se uma questão de gênero importante. ${ }^{13}$ 


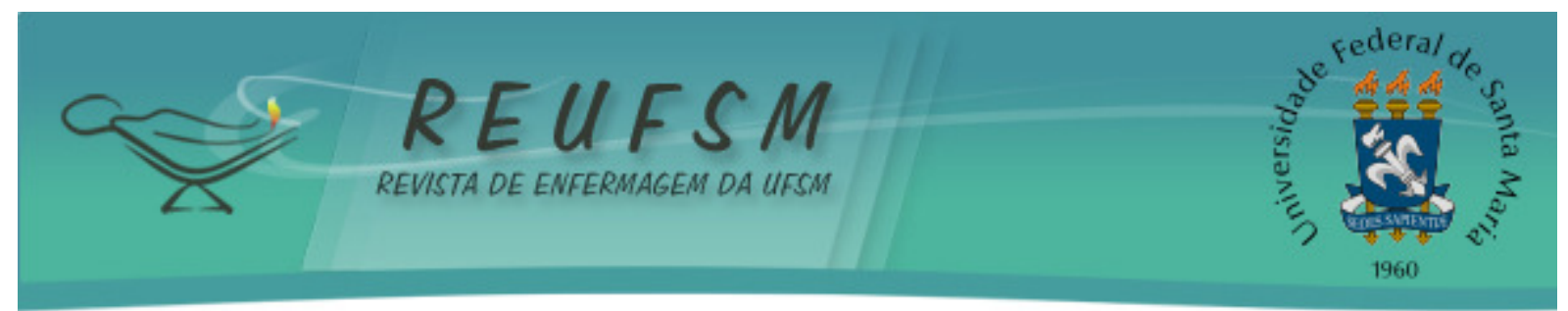

Confirmando, estudo realizado com 11 professores do estado de Goiás cujo objetivo foi identificar os fatores que geravam satisfação e insatisfação com a docência identificou que 10 docentes eram do sexo feminino e idade entre 29 e 59 anos. O tempo de docência variou entre um e 40 anos, sendo que $27,3 \%$ tinha entre um e 10 anos de carreira docente e $72,7 \%$ mais de 10 anos. Todos os docentes estavam sob o regime de dedicação exclusiva, sendo 81,8\% doutores e 18,2\% mestres. Os docentes afirmaram (72,7\%) satisfação no trabalho apesar do excesso de trabalho aliado às atividades administrativas, burocráticas, assistenciais, salas de aulas com muitos alunos. ${ }^{1}$

Ainda em relação aos resultados relacionados ao perfil sócio demográfico dos docentes; uma investigação realizada em uma cidade da região do Sul do Brasil com o objetivo investigar a condição de saúde, o estilo de vida e as características de trabalho dos professores municipais obteve como resultados a predominância do sexo feminino, de meia idade, brancos, casados e com dependentes. ${ }^{14}$

Corroborando, em um estudo realizado com objetivo de identificar as condições do trabalho e os motivos de afastamento do docente universitário de enfermagem, observou-se a predominância do sexo feminino $(64,44 \%)$, casadas $(53,3 \%), 64,4 \%$ exerciam outra profissão para aumentar renda familiar e os motivos para afastamentos foram relacionados a problemas musculo-esqueléticos. ${ }^{15}$

Sobre o regime de trabalho, verifica-se que os professores estão imersos em um conflito cotidiano entre o que é exigido, o que desejam e o que realmente é possível fazer diante dos obstáculos, das condições e da organização atual do trabalho. ${ }^{16}$ Além disso, sabe-se que a carga horária dos professores é intensa e demanda grandes períodos de preparo das disciplinas. Este ponto deve ser discutido e repensado, pois há que se remanejarem os horários e se definir os limites entre trabalho e vida. ${ }^{17}$

Parece ser consenso o reconhecimento do mal-estar manifestado pelos professores, causado pelo aumento de exigências e pressões exercidas sobre as atividades a serem desempenhadas. ${ }^{18}$

Confirmando com os achados desta investigação, uma pesquisa realizada com 24 docentes de três universidades da cidade de São Paulo constatou que em relação às atividades de pesquisa, os professores deveriam desenvolver pesquisas por exigência institucional e a divulgar seus resultados em eventos e publicações. Além disso, os professores mencionaram que, por vezes, o tempo era insuficiente para a realização de tais atividades, o que os levava a realizá-las fora do horário de trabalho. ${ }^{19}$ 


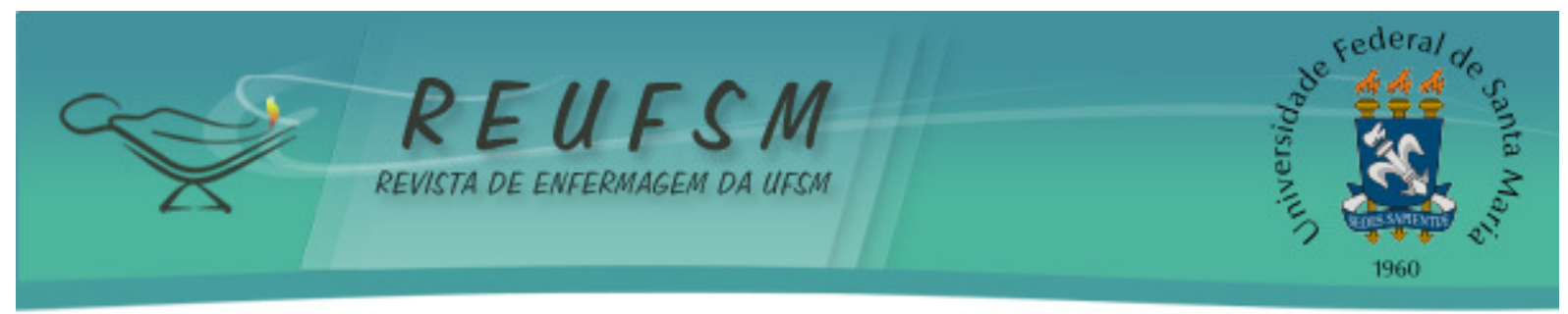

Nesta investigação, os docentes relataram, ainda, que não existem ambientes propícios ao convívio com a comunidade universitária e nem, mesmo, momentos de lazer com colegas de trabalho. Isso foi explicado devido ao fato de haver grande quantidade de atividades a serem realizadas na universidade que, muitas vezes, ficam inacabadas o que faz com que os docentes levem atividades para serem realizadas em casa.

Em relação a esse achado, estudo verificou que as condições de trabalho têm envolvido modelos de produção e prestação de serviços em ritmo acelerado e de modo intensificado. Este modelo determina o aumento da produtividade por combinação do ritmo, da carga de responsabilidade e da redução dos intervalos de descanso na jornada de trabalho, que desencadeia a tendência progressiva de riscos ocupacionais e pode apresentar efeitos crônicos à saúde dos trabalhadores. ${ }^{20}$

Quanto ao afastamento por doenças e AT, estudos demonstram que o trabalho é caracterizado por fatores de riscos que, a depender da estrutura presente em seu ambiente e forma de organização, pode favorecer o aparecimento de doença a quem trabalha. ${ }^{21}$ Por isso, o equilíbrio entre o indivíduo, o ambiente e a satisfação no trabalho faz-se necessário, pois, a interação negativa do sujeito e as condições ambientais do seu trabalho podem ocasionar ansiedade e insatisfação, levando ao acometimento de patologias. ${ }^{22}$

Essas enfermidades estão diretamente conectadas ao trabalho dos educadores, especialmente quando se trata de docentes da área de saúde, em que verifica-se uma alta taxa de estresse, como: a síndrome de Burnout, doenças psicossociais, dano emocional, além de hipertensão arterial, hipotireoidismo e a Diabetes Mellitus. ${ }^{23}$

Levando em conta o âmbito internacional, estudo descritivo realizado com professores de 9 escolas de ensino fundamental da República da Macedônia, com o objetivo de investigar o estresse e sua relação com as características demográficas e laborais, teve como resultados os mais altos níveis de estresse no trabalho estiveram relacionados a ausência de recursos financeiros para fazer trabalhos, pesquisa e acesso a formação continuada. ${ }^{24}$

Sobre os itens analisados da Escala de Estilo de Vida Fantástico, sabe-se que o estilo de vida é compreendido como uma forma cultural e social de viver, abrange hábitos e condutas capazes de contribuir de forma positiva ou negativa à saúde e, também, pode refletir no aumento das taxas de morbidade e mortalidade. O sedentarismo, o consumo de álcool e fumo e a dieta inadequada são 


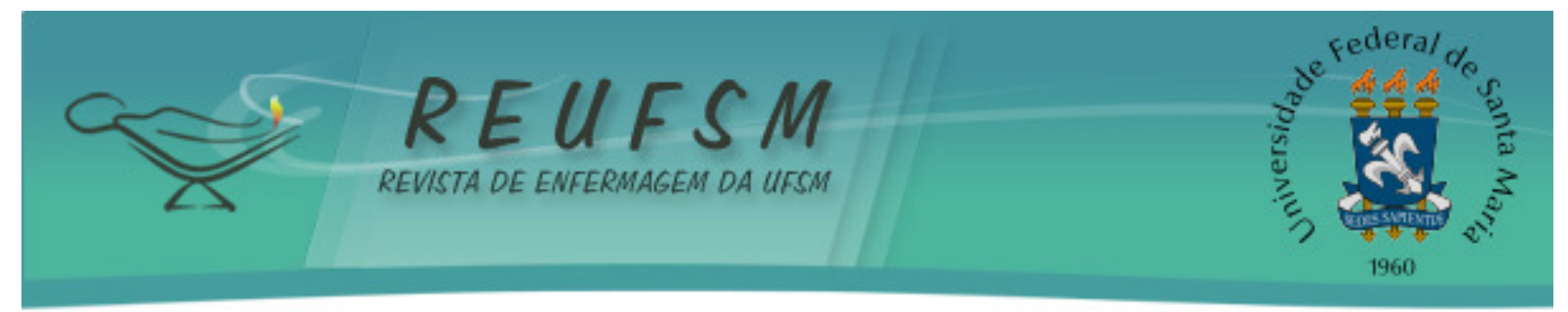

exemplos de hábitos que podem aumentar o risco de morte e agravo à saúde; por outro lado, hábitos como a dieta equilibrada e atividade física regular são considerados protetores. ${ }^{25}$

O estilo de vida pode não afetar a produtividade do trabalho, mas, juntamente com o nível de satisfação laboral, pode interferir no cotidiano dos trabalhadores. Estudos ${ }^{26}$ apontam que a relação de causalidade entre satisfação laboral e cotidiano de trabalho indicam que profissionais motivados e possuidores de um estilo de vida saudável apresentam fatores importantes para a manutenção da saúde, ${ }^{26}$ ou seja, o estilo de vida repercute diretamente na saúde dos indivíduos.

Em relação ao estilo de vida, estudo constatou que a maior parte dos professores da rede estadual do Paraná adotaram comportamentos positivos quanto ao estilo de vida. No entanto, comportamentos negativos foram relatados nos componentes relacionados a nutrição, atividade física e controle do estresse. Os professores mais experientes adotaram comportamentos menos positivos em relação à atividade física do que os professores novatos. O estudo recomenda o desenvolvimento de políticas institucionais de valorização da profissão docente e que proporcionem o aumento do nível de satisfação no ambiente acadêmico ao longo da carreira docente ${ }^{27}$ e, consequentemente, no estilo de vida saudável. Cabe ressaltar que, nos achados do presente estudo, 58,3\% dos docentes indicaram seu Estilo de Vida como "Muito Bom", o que está de acordo com a literatura.

Estudo cujo objetivo foi avaliar o estilo de vida relacionado à saúde de professores de escolas públicas estaduais de Uruguaiana identificou que quanto maior a carga horária de trabalho do professor, mais negativo é seu estilo de vida. A grande quantidade de tarefas acadêmicas, falta de tempo para atividades físicas faz com que optem por alimentos industrializados cujo preparo é mais rápido, porém menos saudáveis, além do tempo reduzido para atividades de lazer e relaxamento que eleva o aparecimento de doenças. ${ }^{28}$

É perceptível, a relevância que o trabalho promove nas vidas dos trabalhadores. Há, contudo uma clara contradição em relação à atividade laboral: alguns veem como algo difícil, de extrema importância para que tenha satisfeitas suas necessidades humanas básicas. Porém, para outros, o trabalho é em sua primeira estância algo desafiador, que dá sentido à vida, definidor de responsabilidades e crescimento ${ }^{29}$ demonstrando sua influência no estilo de vida de cada trabalhador. 


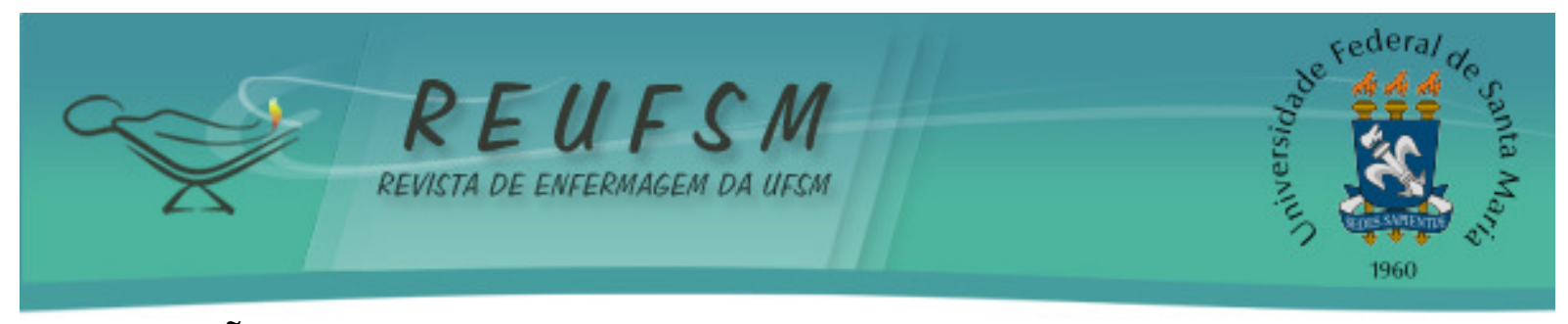

\section{CONCLUSÃO}

Essa pesquisa teve como resultado central a sobrecarga de trabalho docente. Os docentes pesquisados apresentaram jornada de trabalho superior a dez horas, devido às aulas teóricas e práticas, correção de provas em casa, atividades administrativas, porém, estavam realizados na profissão e sua maioria apresentou estilo de vida muito bom. Em relação a saúde, alguns docentes adoeceram devido ao trabalho e a maioria não se sente curada. Destacaram a necessidade da implementação de ambientes de convívio e lazer durante a jornada de trabalho, para proporcionar um ambiente mais saudável e acolhedor tanto aos docentes quanto aos alunos.

A saúde e o trabalho estão interligados. Promover e incentivar um estilo de vida saudável ao trabalhador docente e propiciar condições para tal no ambiente laboral são condições fundamentais, pois, relaciona-se diretamente com a qualidade do ensino proporcionada e a saúde do trabalhador docente.

Este estudo visou considerar a lacuna existente na literatura de estudos que contemplam a saúde do docente de ensino superior. A presente investigação, não pretende realizar generalizações a respeito do estilo de vida e saúde de docentes do ensino superior, porém, cabe ressaltar que, apesar do estudo ter sido realizado com docentes de cursos de saúde de um único Campus de uma Instituição de Ensino Superior Pública, os resultados explanados ao longo do artigo corroboram com os achados da literatura.

Portanto, é necessário ressaltar a necessidade de criação de políticas públicas de saúde que visem a promoção à saúde no ambiente universitário e a produção de novos estudos acerca das alterações de saúde docente e suas repercussões no estilo de vida.

\section{REFERÊNCIAS}

1. Cardoso CGLV, Costa NMSC. Fatores de satisfação e insatisfação profissional de docentes de nutrição. Ciênc Saúde Coletiva [Internet]. 2016 [acesso em 2017 jul 8];21(8):2357-64. Disponível

em: http://www.scielo.br/scielo.php?script=sci_arttext\&pid=S141381232016000802357\&lng=en.

2. Lago RR, Cunha BS, Borges MFSO. Percepção do trabalho docente em uma universidade da região norte do Brasil. Trab. Educ. Saúde, Rio de Janeiro. 2015;13(2):429-50.

3. Oliveira Filho A, Netto-Oliveira ER, Oliveira AAB. Qualidade de vida e fatores de risco de professores universitários. Rev Educ Fís/UEM [Internet]. 2012 [acesso em 2017 jul 8];23(1):57-67.

Disponível

em:

http://www.periodicos.uem.br/ojs/index.php/RevEducFis/article/view/10468/9330. 


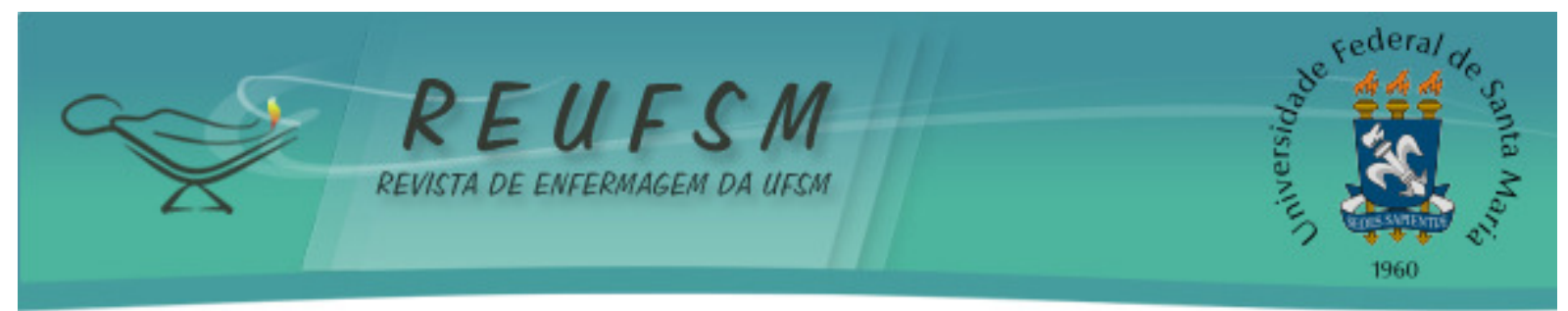

4. Giannin SP, Latorr MDOR, Fischer FM, Ghirardi AC, Ferreira LP. Teachers' voice disorders and loss of work ability: a case-control study. J Voice [Internet]. 2015 [acesso em 2017 jul 8];29(2):209-17. Disponível em: WWW.ncbi.nlm.nih.gov/pubmed/25499521.

5. Naghieh A, Montgomery $\mathrm{P}$, Bonell CP, Thompson M, Aber JL. Organizational interventions for improving wellbeing and reducing work-related stress in teachers. Cochrane Database Syst Rev [Internet]. 2015 [acesso em 2017 jul 8];8(4). Disponível em: onlinelibrary.wiley.com/doi/10.1002/14651858.CD010306/pdf.

6. Vale SCP, Aguillera F. Estresse dos professores de ensino fundamental em escolas públicas. Rev Psicologia, Diversidade e Saúde, Salvador [Internet]. 2016 [acesso em 2017 jul 9];5(1):86-94.

Disponível

em:

HTTPS://www5.bahiana.edu.br/index.php/psicologia/article/download/712/595.

7. Santos MN, Marques AC. Condições de saúde, estilo de vida e características de trabalho de professores de uma cidade do sul do Brasil. Ciênc Saúde Colet [Internet]. 2013 [acesso em 2017 jul 9];18(3):837-46. Disponível em: www.scielo.br/pdf/csc/v18n3/29.pdf.

8. Añez CRR, Reis RS, Petroski EL. Versão brasileira do questionário "Estilo de Vida Fantástico: tradução e validação para adultos jovens. Arq Bras Cardio. 2008;91(2):102-9.

9. Brasil. Conselho Nacional de Saúde. Resolução CNS nº 466, de 12 de dezembro de 2012. Aprovar as seguintes diretrizes e normas regulamentadoras de pesquisas envolvendo seres humanos [Internet]. 2012 [acesso em 2017 jul 11]. Disponível em: http://bvsms.saude.gov.br/bvs/saudelegis/cns/2013/res0466_12_12_2012.html.

10. Landis JR, Koch GG. The measurement of observer agrément for categorial data. Biometrics [Internet]. 1977 [acesso em 2017 out 19];33(1):159-74. Disponível em: https://www.ncbi.nlm.nih.gov/pubmed/843571

11. Carvalho JF, Martins EPT, Lúcio L, Papandréa PJ. Qualidade de vida no trabalho e fatores motivacionais dos colaboradores nas organizações, educação em foco [Internet]. 2013[acesso em 2017 jul 10];9(7):21-31. Disponível em: http://unifia.edu.br/revista_eletronica/revistas/gestao_foco/artigos/ano2013/setembro/qualidad e_motivacao.pdf.

12. Cardoso JP, Araujo TM, Carvalho FM, Oliveira NF, Reis EJFB. Aspectos psicossociais do trabalho e dor musculoesquelética em professores. Cad Saúde Pública [Internet]. 2011 [acesso em 2017 jul 10];27(8):1498-506. Disponível em: https://repositorio.ufba.br/ri/handle/ri/18109.

13. Souza KR, Brito JC. Sindicalismo, condições de trabalho e saúde: a perspectiva dos profissionais da educação do Rio de Janeiro. Ciênc Saúde Colet [Internet]. 2012 [acesso em 2017 jul 9];17(2):379-88. Disponível em: www.scielo.br/pdf/csc/v17n2/a12v17n2.

14. Santos MN, Marques AC. Condições de saúde, estilo de vida e características de trabalho de professores de uma cidade do sul do Brasil. Ciênc Saúde Colet [Internet]. 2013 [acesso em 2017 jul 9];18(3):837-46. Disponível em: www.scielo.br/pdf/csc/v18n3/29.pdf.

15. Oliveira JM, Santos PF, Feliciano RG, Assis MM, Cortez AE, Valente GSC. Riscos e doenças ocupacionais do docente universitário de enfermagem: implicações na saúde do trabalhador. Rev 


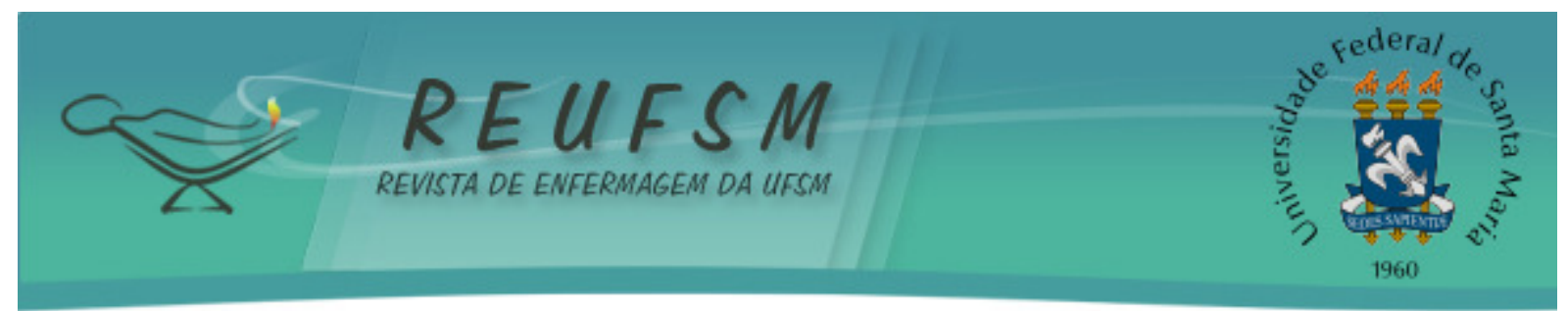

Pesqui Cuid Fundam [Internet]. 2013 [acesso 2017 jul 9];5(1):3267-75. Disponível em: http://www.seer.unirio.br/index.php/cuidadofundamental/article/view/1903/pdf_684.

16. Vieira SRS. Sofrimento psíquico e trabalho. Rev Latinoam Psicopatol Fundam. 2014;17 (1):114-24.

17. Koetz L, Rempel C, Perico E. Qualidade de vida de professores de Instituições de Ensino Superior Comunitárias do Rio Grande do Sul. Ciênc Saúde Colet [Internet]. 2013 [acesso em 2017 jul 10];18(4):1019-1028. Disponível em: http://www.scielo.br/pdf/csc/v18n4/15.pdf.

18. Alves GM, Gonçalves TNR, Azevedo NR. Satisfação e situação profissional: um estudo com professores nos primeiros anos de carreira. Educ Pesqui [Internet]. 2014 [acesso em 2017 jul 9],40 (abr-jun). Disponível em: http://www.scielo.br/pdf/ep/v40n2/aop1213.pdf.

19. Leonello VM, Oliveira MAC. Educação superior em Enfermagem: o processo de trabalho docente em diferentes contextos institucionais. Rev Esc Enferm USP [Internet]. 2014 [acesso em 2017 jul 9];48(6):1093-102. Disponível em: www.scielo.br/pdf/reeusp/v48n6/pt_00806234-reeusp-48-06-1093.pdf.

20. Hilleshein LF, Lautert L. Capacidade para o trabalho, característica sócio demográficas e laborais de enfermeiros de um hospital universitário. Rev Latinoam Enferm [Internet]. 2013 [acesso em 2017 jul 9];20(3):[8 telas]. Disponível em: WWW.scielo.br/pdf/rlae/v20n3/pt_a13v20n3.pdf.

21. Caran VCS, Freitas FCT, Alves LM, Pedrão LJ, Robazzi MLCC. Riscos ocupacionais, psicossociais e sua repercussão na saúde de docentes universitários. Rev Enferm UERJ [Internet]. 2011 [acesso 2017 jul 9];19(2):255-61. Disponível em: www.facenf.uerj.br/v19n2/v19n2a14.pdf.

22. Teixeira JRB, Boery EM, Casotti CA, Araújo TM, Pereira R, Ribeiro IJS, et al. Associação entre aspectos psicossociais do trabalho e qualidade de vida de mototaxistas. Cad Saúde Públ [Internet]. 2015 [acesso 2017 jul 9];31(1):97-110. Disponível em: WWW.scielosp.org/pdf/csp/v31n1/0102-311X-csp-31-01-00097.pdf.

23. Terra FS, Secco IAO, Robazzi MLCC. Perfil dos docentes de cursos de graduação em Enfermagem de universidades públicas e privadas. Rev Enferm UERJ [Internet]. 2011 [acesso em 2017 jul 9];19(1):26-33. Disponível em: www.facenf.uerj.br/v19n1/v19n1a05.pdf.

24. Agai-Demjaha T, Minov J, Stoleski S, Zafirova B. Stress causing factors among teachers in elementary schools and their relationship with demographic and job characteristics. Macedonian J Med Sciences [Internet]. 2015 [acesso 2016 jun 9];3(3):493-9. Disponível em: WWW.id-press.eu/mjms/article/view/oamjms.2015.077.

25. Ferreira DKS, Bonfim C, Augusto LGS. Fatores associados ao estilo de vida de policiais militares. Ciênc Saúde Colet [Internet]. 2011 [acesso 2016 jun 8];16(8):3403-12. Disponível em: www.scielo.br/pdf/csc/v16n8/a07v16n8.pdf.

26. Both J, Nascimento JV. Intervenção profissional na educação física escolar: considerações sobre o trabalho docente. Movimento [Internet]. 2009 [acesso 2017 jun 8];15(2):169-86. Disponível em: www.seer.ufrgs.br/Movimento/article/download/3046/5140.

27. Moreira HR, Nascimento JV, Sonoo CN, Both J. Qualidade de vida no trabalho e perfil do estilo de vida individual de professores de Educação Física ao longo da carreira docente. 


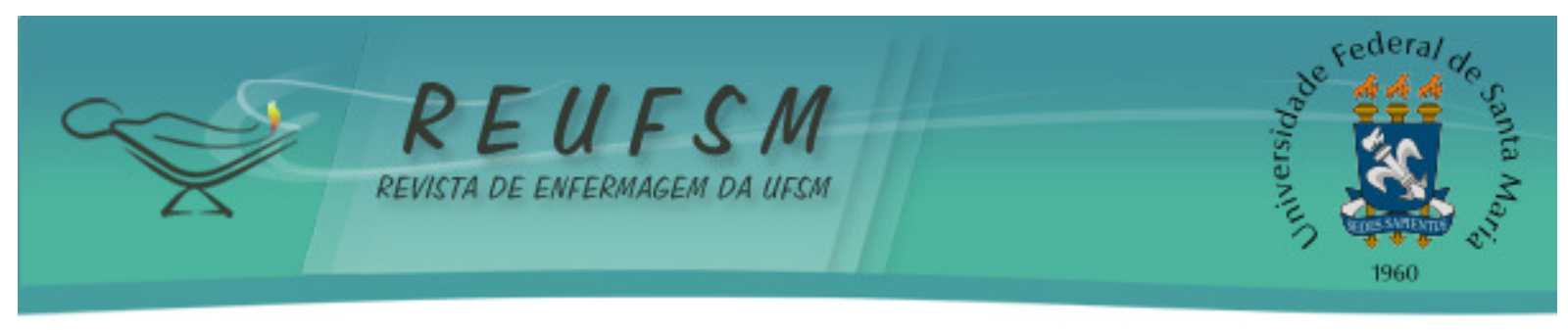

Motriz [Internet]. 2010 [acesso 2017 out 4];16(4):900-12. Disponível em http://www.scielo.br/pdf/motriz/v16n4/a10v16n4.pdf

28.Coutinho RX, Santos WM, Soares MC, Santos CM, Folmer V, Puntel RL. Perfil do estilo de vida relacionado à saúde de professores. EFDeportes [Internet]. 2012 [acesso 2017 out 4];17(168). Disponível em: http://www.efdeportes.com/efd168/estilo-de-vida-relacionado-aprofessores.htm

29.Freitas EO, Nogueira RS, Stekel LMC, Bublitz S, Kirchhol R, Guido LA. Perfil de pacientes com doença arterial coronariana submetidos ao cateterismo cardíaco. Rev Enferm UFSM. 2013;3(Esp.):679-88.

Data de submissão: 08/11/2016

Data de aceite: 21/09/2017

Autor correspondente: Renata Cristina da Penha Silveira

Endereço: Rua Sebastião Gonçalves Coelho, 400. Bairro: Chanadour. Divinópolis (MG), Brasil.CEP: 35501-296.

Email: renatasilveira@ufsj.edu.br 